\title{
Deserción a nivel universitario
}

\author{
Mario Castillo Sánchez
}

\section{Introducción}

Las características generales de nuestro país ofrecen diversas oportunidades para que la población se sienta motivada a alcanzar una mejor calidad de vida, por lo que año a año son miles los estudiantes que inundan universidades con el objetivo de mejorar su condición. Pero también es cierto que muchos abandonan sus estudios sin haber alcanzado su ideal. Esta situación genera polémica y es un tema que merece su estudio y análisis.

Cuando un joven se retira de la universidad sin haber alcanzado un título, cuando no alcanza los niveles más altos de educación está en desventaja para competir en un mercado laboral cada vez más competitivo.

La deserción de los estudios es un fenómeno que afecta a todas las universidades, sin embargo, cuando alcanza niveles altos, la hacen merecedora de un interés primordial dentro del conjunto de problemas que existen en el ámbito universitario.

Es evidente la pérdida de recursos económicos y su efecto negativo sobre la formación de capital humano, así 
como el desaliento y el sentimiento de frustración que produce en los estudiantes. Sin embargo, a pesar de su importancia, se han realizado pocas investigaciones para apreciar el estudio de este problema, sus causas y las posibles soluciones. A este respecto, existen pocos datos detallados sobre la deserción. Por esta razón, se considera necesario investigar sobre los índices de deserción, y las características relacionadas con ésta, tanto para el desarrollo de estrate. gias que disminuyan el problema, como también para ser considerados en la planificación del desarrollo académico de las instituciones.

El problema de la deserción no es solamente académico sino también familiar, pues los recursos económicos son dirigidos a la atención de un conjunto de necesidades, dentro de los cuales se incluye el gasto en estudio. Las decisiones que la familia tome al respecto son vitales, pues permiten estimular al estudiante a posponer el estudio, o bien continuarlo.

En el caso de familias de escasos recursos, la decisión de continuar los estudios representa un verdadero sacrificio. Así, el papel de la universidad es fundamental y tiene la responsabilidad de ofrecer instancias eficientes para que el estudiante pueda exponer sus problemas, recibir ayuda no solo económica sino asesoría apropiada para lograr el éxito en sus estudios.

La deserción no afecta solamente al individuo, sino a la sociedad en general. La universidad debe proveer los recursos humanos necesarios para el desarrollo nacional, transmitir el patrimonio cultural, constituir el eje del sistema científico tecnológico, difundir la cultura, realizar una labor de crítica social y contribuir al desarrollo del país.

Los costos de la educación son muy altos y la inversión que se hace en este campo debe recuperarse a través de la formación de un tipo de ciudadano cada vez más apto y 
capaz, por esto la deserción estudiantil debe ser objeto de mayor preocupación e interés, ya que, sus implicaciones para llevar a cabo los objetivos de la universidad son de suma importancia.

Las causas de la deserción podrían estar determinadas por condiciones objetivas, particularmente características socioeconómicas, psicológicas, culturales propias del estudiante y del entorno en el cual se desenvuelve; así como factores propios de la institución y del sistema de enseñanza y aprendizaje que, de manera directa o indirecta, interactúan para que se produzca el abandono.

Por tanto, la identificación y el correspondiente análisis de las causas y las consecuencias de la deserción, permiten generar acciones dentro de la universidad, dirigidas a disminuir los niveles de deserción existentes y con ello, favorecer la formación de futuros profesionales que contribuyan al desarrollo del país.

En el caso de las universidades es el departamento de Asuntos Estudiantiles o de Vida Estudiantil, quien se encarga del desarrollo de programas preventivos, educativos y de asistencia. Estos van dirigidos a estudiantes que poseen aptitudes académicas e interés por cursar una carrera universitaria, pero que, por su difícil situación socioeconómica, no lo pueden hacer por sus propios medios.

Para hacer más efectivos los programas de bienestar estudiantil es importante conocer las probabilidades de deserción que tienen los estudiantes que matriculan en la universidad y las razones de tal deserción. También es importante conocer la asociación que puedan tener algunas variables sociodemográficas con la deserción estudiantil, para poder determinar los subgrupos que los programas deberían establecer como prioritarios. 


\section{Situación actual y tendencias de la educación superior}

Una de las situaciones que tuvo y que tiene que enfrentar la educación superior costarricense fue el crecimiento demográfico de los años cincuenta. A partir de 1967 se inicia la incorporación masiva de estudiantes a la universidad, con altas tasas anuales de crecimiento. Costa Rica pasó por altos períodos de crecimiento demográfico en los años 50 e inicios de los 60, lo que vino a afectar la población en edad de entrada a las universidades. De 1967 a 1981, se incrementó más de siete veces la cantidad de estudiantes en las universidades costarricenses, pasando de 6000 a 54000 , crecimiento que significó un incremento del $790 \%$ y distorsionó totalmente las tendencias históricas.

El "baby boom" de 1955 a 1965 implica que como promedio, anualmente se estén incorporando, desde 1976 (al llegar a una edad de 21 años) más de 56000 ciudadanos a la vida laboral o a la educación superior.

Es importante indicar que, algunos investigadores consignan un nuevo boom demográfico entre 1975 y 1985 , está población ejercerá presión en los años venideros por un cupo en la educación superior y, por ende, una mayor calidad de educación, es en este aspecto donde se debe dar la competencia entre universidades públicas y privadas. (Ruiz, 2001)

Desde la aparición del sistema educativo superior, obtener un título universitario se transformó en un gran reto para la familia costarricense y desde el punto de vista social, se gestó un mecanismo de promoción social o movilidad social que tendría consecuencias para el desarrollo equilibrado de la sociedad.

Este desafío por alcanzar un título universitario, llega también al sector femenino, y se produce un fenómeno de suma importancia para nuestro país: la incorporación de la 
mujer al sistema educativo universitario, que se inicia a partir de los años setenta.

Costa Rica no escapa a la crisis económica que ha tenido prácticamente toda la humanidad y ya para la década de los ochenta se da una disminución en el presupuesto destinado para educación en general en el país. Si bien es cierto que según este indicador Costa Rica aún se mantiene en un sitio de privilegio en el área latinoamericana, también es cierto que ha perdido terreno y que presenta una tendencia hacia la declinación.

El mundo se dirige hacia la globalización en muchos sentidos, pero serán las comunicaciones y la penetración masiva de productos y servicios, las que con más fuerza modificarán valores, modos de vida y costumbres. Se sostiene que la tendencia será hacia una pérdida cada vez mayor de las diferencias culturales.

Jofré (1998) argumenta que, el mundo camina hacia la estandarización, a imponer valores de sociedades más industrializadas, hacia la universalización de los valores. El desarrollo de los medios de comunicación, la globalización de los mercados, la pérdida de relevancia de las fronteras, irán acabando pausadamente las identidades culturales. Lo que antes llevaba siglos, ahora se produce en unas pocas décadas.

En cuanto a este tema, la universidad no solo tiene la gran oportunidad de establecer el engranaje necesario para rescatar las diferencias culturales y transformar esto en una orientación que desvie en algún modo la tendencia estandarizadora, sino que tiene también la ocasión de recuperar su papel en la sociedad.

En este sentido, la universidad tiene que ser protagonista, fundamentada en su misión de conservar y transmitir la cultura, la universidad no puede mostrarse indiferente en relación con las costumbres, ideas, expresiones artísticas, y los valores que han fortalecido a la sociedad. 
En este aspecto, Angel Ruiz (2001) considera que como efecto de la globalización, la educación en general y, sobre todo, la educación superior, tiene que mejorar la calidad de la educación, no solo en la formación de sus graduados, sino también en un manejo eficaz de los recursos con que cuenta, así como contextualizar sus carreras dando énfasis al tiempo destinado para graduarse, al manejo eficaz de sus recursos, a la pertinencia del currículo, y a la calidad y formación de sus docentes.

Un fenómeno interesante, y que cabe mencionar, es que el nuevo boom demográfico, cambiará las condiciones económicas del país en las primeras dos décadas del presente siglo, según el Dr. Rosero, la población del primer boom no está aún vieja, trabaja, produce y ahorra y, por otro lado, la generación del segundo boom se está incorporando a la producción del país, por lo que tiende a disminuir el número de dependientes por familia. Sin embargo, para el año 2020 la población del primer boom estará ya en la tercera edad y se terminará esta etapa favorable económicamente para el país. Esto indica que estos primeros años de siglo serán determinantes para el progreso de Costa Rica. Y es aquí donde las universidades públicas y privadas tienen una gran responsabilidad aunque no exclusiva, para formar esta nueva generación que ha vivido tiempos difíciles económicos y de estilo de vida. (Ruiz, 2001).

Según proyecciones de la UNESCO en el nuevo siglo, a lo sumo el $10 \%$ de la población de los países en desarrollo estará matriculada en la educación superior, por lo que la universidad tiene el gran desafío de lograr una mayor equidad en el acceso sin que esto repercuta en la calidad.

En el presente siglo no se podría hablar de una educación universal, de un modelo único, sino que hay que hablar de modelos educativos que respondan a diferentes grupos sociales, diferentes también en sus niveles de desarrollo y en sus características culturales. 
El conocimiento, o mejor dicho la información adquiere cada vez más importancia y es más necesaria en relación con la materia prima, el trabajo y otros recursos. El conocimiento es la nueva ventaja comparativa de las naciones. El factor que decidirá el destino de las naciones en la economía global será el nivel educativo de sus poblaciones, los nuevos empleos exigen conocimientos, imaginación e inteligencia.

Por tal razón es insuficiente que los sistemas de educación brinden solamente los conocimientos técnicos productivos, deben promover conjuntamente con el conocimiento técnico, el desarrollo de la inteligencia y la creatividad.

Otro fenómeno que influye en los sistemas de educación superior es la creciente participación de la mujer, que requiere un cambio en las estructuras actuales y una actualización de los currículos, igualmente los sectores de trabajadores adultos influyen en el currículum pues deben recalificar sus competencias laborales.

Sin embargo, no podemos obviar que, con las nuevas tecnologías la forma de ver el mundo varía rápidamente: aparecen nuevas representaciones mentales, nuevas experiencias vivenciales, acceso a una cantidad inmensa de información y nuevos entornos de aprendizaje.

La universidad debe preparar al hombre del nuevo siglo, para que sea capaz de asimilar este bombardeo de cambios tecnológicos. No se trata de formar un estudiante pasivo, consumidor de información, sino, un estudiante activo y reflexivo, por tanto, un constructor de su propio conocimiento.

La Universidad se constituye, hoy más que nunca, en un instrumento indispensable para nuestra sociedad, que demanda una preparación de diferentes cuadros profesionales, diferenciados por su presenciabilidad en el tiempo y en el espacio y, sin que pierdan los niveles de producción socioeconómicamente alcanzados. 
Con la globalización, la base de acción son los requerimientos del mercado y, la eficiencia productiva es determinante para poder competir a nivel internacional. Esta situación envuelve grandes conflictos y uno de ellos es la repercusión sobre el mercado laboral.

Estos desafíos establecen la necesidad de hacer ajustes en las instancias educativas, para que los trabajadores estén en condiciones de incorporarse adecuadamente al mercado laboral. Esto no es sinónimo de hacer "robots" perfectamente adaptables a dichos mercados. Es en este aspecto, que el reto de la educación se hace más difícil, porque el sistema educativo debe ser flexible para el cambio, pero lo adecuadamente fundamentado y consolidado como

En este sentido, la universidad debe tener presente que la educación debe ser continua, que todo estudiante aún después de graduado deberá regresar a los recintos universitarios para actualizarse.

\section{Conceptualización de la deserción}

El término "desertor" se aplica a quien abandona una actividad en forma imprevista y deja inconclusos los objetivos iniciales planteados. En el campo educativo la deserción estudiantil se entiende como un fenómeno que se caracteriza por el abandono definitivo de la asistencia a la institución educativa.

En forma general, la deserción educativa se refiere a una manifestación de conducta, que podría resultar de la interacción de una serie de características o variables, que influyen en la decisión por parte del educando, en este caso específico de un estudiante universitario, por interrumpir sus actividades académicas

La deserción es el resultado de una combinación de factores, e implica un costo social importante, en el sentido 
de que se da una inversión durante un tiempo determinado, para formar a un individuo, que no culmina su carrera y que por lo tanto no logra los objetivos que en un inicio se había planteado. Se considera deserción, por lo tanto, el abandono del sistema educativo por parte del alumno.

La deserción se puede considerar como un parámetro primordial para medir el desarrollo educativo de la población y la eficiencia del sistema educativo de un país, siendo en la actualidad un problema al que se enfrentan miles de estudiantes.

A veces resulta difícil determinar cuándo debe o puede considerarse que un estudiante inscrito ha abảndonado sus estudios, en un curso o en una carrera. La situación se torna aún más compleja cuando los alumnos que abandonan sus estudios pueden en cualquier momento volver a la institución universitaria. Es decir, dejan uno o más periodos académicos sin estudiar y luego vuelven. Esta complicación dificulta mucho la evaluación e interpretación de las cifras sobre este tema, y no permite en múltiples oportunidades, comparaciones o conclusiones sólidas rigurosas.

Desde una perspectiva psicosocial, la deserción genera un fuerte impacto en el sujeto que hace abandono de sus estudios. Por ejemplo, produce sentimientos de frustración, que con el paso del tiempo pueden conducir a un sentimiento de fracaso.

Generalmente, cuando las personas encuentran una serie de limitaciones económicas para adquirir los bienes y servicios que desean, asumen como causa de tal hecho la carencia de una preparación académica.

Factores asociados con la deserción

Al mencionar la deserción estudiantil surge una serie de elementos que están asociados con la determinación que 
toma un individuo al abandonar sus estudios, algunas de las características a considerar son inherentes al estudiante (género, edad), otras se encuentran relacionadas con el entorno del individuo; en este apartado se analizan los atributos individuales que podrían tener alguna relación con el abandono de los estudios y se revisan los factores motivacionales que inciden en la decisión de abandono, luego se analizan los factores socioeconómicos y académicos del alumno y finalmente, los factores institucionales que afectan al individuo, propiamente los elementos administrativos que de alguna manera inciden en la deserción estudiantil.

Si bien la decisión de abandonar los estudios universitarios es el resultado de múltiples factores, algunas investigaciones han encontrado relaciones interesantes entre la deserción y algunas características personales de los estudiantes, es así como el género es un atributo considerado en investigaciones sobre el abandono. Woodley y Parlett, citados por Granados (1992) en un estudio realizado en la Open University, encontraron que la tasa de abandono en los estudiantes de género masculino es más elevada que en los estudiantes femeninos. Sin embargo, en una investigación realizada en la Universidad Nacional de Educación a Distancia, en España, se determinó que el género no produce diferencias significativas a la hora de abandonar los estudios y concluyeron que el hecho de realizar estudios en una universidad a distancia, puede disminuir las diferencias inherentes a cada género (maternidad, trabajo, estado civil). Igualmente, en una investigación realizada en Argentina, se determinó que el riesgo de abandono para los hombres era 1,36 veces mayor que el de las mujeres. Es decir, que en una institución de educación presencial el género sí produce diferencias significativas en cuanto al índice de deserción.

En lo referente a la edad de los estudiantes, los resultados indican que a mayor edad es menor la tasa de abandono, se 
indica que el aprender a distancia requiere cierto grado de madurez, por esta razón la Open University después de un estudio sobre rendimiento de los estudiantes decidió establecer una edad mínima de 21 años como requisito para ingresar.

Es de esperar que los estudiantes jóvenes, y que vienen de un sistema tradicional de enseñanza, no tengan la madurez necesaria ni la autodisciplina para luchar contra la sensación de aislamiento que se da en una educación universitaria, por esto, el nivel de deserción entre los estudiantes más jóvenes es probablemente mucho mayor que entre los de más edad. En el estudio realizado en la Open University, se encontró que la mayor tasa de abandono corresponde a estudiantes con edades entre 21 y 24 años y entre los 50 y 59 años.

En la Universidad Nacional de Educación a Distancia, en España, se encontró una relación significativa entre edad y rendimiento, resultando que los estudiantes con mayor grado de permanencia en la Universidad eran los de 30 a 39 años, y la tasa iba descendiendo según se aumentaba o disminuía la edad, se ubicó el mayor índice de abandono entre los de 21-24 años.

Otro aspecto que determina la situación de una persona es su estado civil, si bien es cierto esta situación está relacionada con la edad del estudiante, en algunas investigaciones se ha determinado que el cambio de estado civil incide significativamente en la conducta de abandono, especialmente en la mujeres. García Aretio (1987) determinó que si bien el estado civil está muy relacionado con la edad de los estudiantes, se puede observar que los estudiantes casados y con hijos presentan las tasas mayores de abandono.

Otro elemento que esta muy relacionado con la edad y con el estado civil es el número de hijos, y a la vez ésta implícitamente relacionado con el abandono de los estudios, 
pues en la investigación realizada en la Open University se determinó que el $77 \%$ de los que desertaban de esa universidad lo hacían por razones familiares.

Por tanto, es importante saber el estado civil del estudiante y tener una idea sobre el número de miembros que constituyen la familia, pues esto puede orientar sobre algunos condicionantes y responsabilidades que pueden tener una influencia significativa sobre la decisión de abandonar los estudios universitarios. Al respecto, García Aretio (1987) determinó que los estudiantes con dos o tres hijos tenían las mayores tasas de éxito.

En lo que se refiere a la situación laboral de los estudiantes, es un hecho que las obligaciones ajenas al estudio demandan tiempo y responsabilidades especiales, hay evidencia de que gran cantidad de estudiantes que abandonan la universidad, lo hacen por carecer del tiempo suficiente para poder resolver las demandas de su trabajo y de sus estudios, así en la Open University, se determinó que la tasa de abandono es más alta entre los alumnos que tienen ocupaciones laborales.

Por su parte, García Aretio (1987) en su estudio determinó que más del $60 \%$ de los que abandonaron la universidad lo hacían por razones laborales, dentro de las que se pueden mencionar, incremento en las horas de trabajo, horario laboral irregular e inclusive cambio de trabajo. Referente a este aspecto, se ha determinado que estudiar y trabajar simultáneamente provoca un mayor riesgo de abandono, 3,4 veces mayor con relación a quienes no laboran.

También las características de las condiciones académicas previas al ingreso a la universidad, pueden condicionar las posibilidad de abandono del alumno, al considerar los estudios previos que tiene el estudiante al ingresar a la institución se tiene algún grado de referencia sobre la madurez intelectual, en este sentido en la Open University se 
determinó que a menor nivel académico previo a su matrícula, la probabilidad de abandono era mayor, igual resultado se halló en la Universidad Nacional de Educación a Distancia, en España; los estudiantes con mayor experiencia académica se mantenían por más tiempo dentro de la universidad.

Por otra parte, García Aretio (1987) determinó que los estudiantes con estudios previos de licenciatura eran los que proporcionalmente más abandonaban los estudios en la Universidad Nacional de Educación a Distancia de España; García Aretio opina que quizás dieron por un hecho que sería fácil obtener un segundo título.

Otra situación que esta relacionada directamente con el abandono es la condición académica de los estudiantes, propiamente el rendimiento académico, al respecto se ha comprobado que la obtención de bajas calificaciones es un factor significativo en el abandono posterior de los estudios y que, por tanto, una persona con bajas calificaciones previas es más propensa a abandonar los estudios. Es decir, que los hábitos de estudio, aptitudes y habilidades están directamente relacionados con el rendimiento y posteriormente con el abandono de los estudios. Esto se vincula con el número de materias matriculadas o la carga académica, pues se ha determinado que el número de asignaturas influye significativamente en el abandono y que la tasa de deserción se incrementa en función de la sobrecargada de trabajo académico.

Referente a esto es realmente necesario proporcionar información y orientación a los estudiantes sobre la carga académica recomendable de matricular, considerando las situaciones personales; de trabajo, las obligaciones familiares, los estudios previos y todas las situaciones que puedan interactuar con su estudio.

Otro aspecto que también está relacionado con el abandono en las instituciones educativas, es el lugar de residencia o visto de otra forma la distancia al centro universitario. 
Granados (1992), en la UNED de España y propiamente en los estudiantes de Letras, deduce que los estudiantes procedentes de un medio familiar urbano mantienen conductas de abandono menores a los procedentes del área rural. En esta misma Universidad, García Aretio (1987) opina que si bien la distancia al centro universitario no es un asunto académico, si se puede considerar como un factor a la hora de tomar la decisión de abandonar los estudios.

Dentro de las teorías de la motivación, se ha considerado que el individuo tiene una jerarquía en sus necesidades que van desde el orden fisiológico, hasta la necesidad de saber y se asegura que unas tienen que satisfacerse para que surjan las otras.

Dentro de los aspectos personales que influyen en la decisión de continuar o abandonar los estudios se puede mencionar los siguientes:

- falta de respuesta de la institución educativa a las expectativas del educando, por ejemplo que la carrera que él escogió no llena sus expectativas, tanto intelectuales como económicas, o que la carrera que está cursando, no es la que, desearía cursar si tuviese los medios para hacerlo.

- falta de interés real por el estudio, motivada por una inadecuada orientación vocacional o por ausencia de ésta

- causas psicofísicas, generalmente problemas de aprendizaje que generan en la decisión de no poder seguir estudiando, principalmente por la no adecuación curricular de la institución

- necesidad de satisfacer otras expectativas antes que las educacionales, que el individuo jerarquiza como prioritarias y que pueden tener respuesta en lo económico o lo social. 
- dificultad para asimilar el ambiente universitario, en otras palabras, no se siente bien con sus profesores o amigos, ni con el sistema de enseñanza, generándose así un estado de insatisfacción que en última instancia puede propiciar el abandono de sus estudios.

La motivación realmente es la que alimenta las expectativas presentes y futuras, es la que orienta al individuo a plantearse un conjunto de metas, por las que estará anuente a luchar y superar los obstáculos que se le presentan. Si el móvil de acción satisface plenamente al estudiante, es muy probable que su nivel de motivación lo impulse a continuar con una actitud positiva hacia la institución, y hacia el estudio, y facilita el proceso de adaptación del estudiante a su nuevo medio.

De otro modo, la situación educativa se torna conflictiva si el educando se percibe fuera de ambiente y la permanencia en la institución le provoca sentimientos de conflicto y malestar general. Se ha sostenido, entonces, que el grado de motivación y nivel de adaptación del individuo al medio, determinará en gran medida la permanencia en la institución educativa.

Uno de los aspectos que se considera primordial a la hora de realizar estudios es la situación económica del estudiante. A pesar de que, generalmente, las universidades, por medio de sus oficinas de becas ofrecen ayuda para solventar en algún grado la problemática económica, no cahe duda que la dificultad de nayor peso para algunos alumnos estriba en las necesidades económicas que se enfrenta. Esta dificil situación puede obligarle a tomar, de buenas a primeras, la oportunidad de trabajo que se le presente sin considerar las consecuencias futuras en su preparación académica.

De tal manera, algunos aspectos que influyen en esta decisión son los siguientes: 
a) la necesidad que tiene el estudiante de trabajar para lograr su manutención y posiblemente la de su familia, actitud generada por la imposibilidad económica del grupo familiar para solventar sus gastos de estudio.

b) la inexistencia de una legislación laboral que incentive los logros educativos realizados por la persona.

Referente al estado de salud se ha determinado que es un factor que contribuye a la deserción por lo menos indirectamente, y se refleja cierta correlación entre bajo rendimiento académico y mal estado de salud.

Como se ha mencionado, existen teóricamente diversas causas que propician la decisión de continuar o abandonar los estudios. Una muy importante es el bajo rendimiento académico, el cual puede ser producto de problemas de aprendizaje causado por defectos sensoriales o problemas específicos, la falta de continuidad en la enseñanza, los cambios continuos de centros educativos, la existencia de profesores con deficiencia de conocimientos básicos y didácticos.

El nivel de exigencia académica enfrenta al estudiante universitario a una situación de carácter diferente a la experiencia acumulada del sistema educativo del que procede, ya que los programas y actividades por naturaleza difieren de los que el alumno estuvo acostumbrado durante años.

La exigencia académica a la que se ven sometidos los estudiantes de primer ingreso, pone de manifiesto las posibles deficiencias adquiridas a lo largo de la trayectoria que este sujeto tuvo como ente receptor e inmerso en un sistema educativo defectuoso.

El rendimiento académico que logra el estudiante está asociado a sus fortalezas o deficiencias de tipo educativo y formativo, tales como: expresión escrita, expresión oral, 
serias dificultades en el nivel de comprensión y retención, carencia de conocimientos previos en aspectos relacionados con la realidad circundante, empleo inadecuado de técnicas de estudio, poca experiencia en la presentación de trabajos escritos, entre otras. Para muchos, su debilidad en estas prácticas representa un obstáculo difícil de superar.

El estudiante universitario, y sobre todo el principiante, necesita mucho apoyo familiar, tanto económico como afectivo, pero también necesita el apoyo institucional. Todo individuo enfrentará en el transcurso de la carrera problemas de toda índole, será ahí donde la ayuda familiar y los aspectos administrativos de la institución desempeñarán un papel preponderante en la decisión de continuar o postergar los estudios. Existen factores administrativos que desmotivan en cierta forma al educando, por ejemplo: cuando los horarios de los cursos no son adecuados a las personas que trabajan, cuando se informa mal a los alumnos antes de matricularse, no se orienta adecuadamente a la persona de primer ingreso y cuando se masifica la educación, tratando al estudiante como un número más de carné. Por las características propias de la Educación Superior los elementos administrativos juegan un papel fundamental en el buen desarrollo de la relación Alumno-Universidad.

Tal como se ha expuesto, la decisión de continuar o abandonar los estudios es un fenómeno socioeducativo que está presente en el quehacer diario de una institución educativa universitaria. Muchos son los elementos que determinan que se tome uno u otro camino, entre ellos: situaciones de salud, económicos, de horario de trabajo y estudio, problemas de rendimiento académico, familiares, problemas de traslado a la Universidad, el grupo de compañeros, los programas académicos, los materiales de aprendizaje, la falta de orientación familiar, la influencia ejercida por el personal docente-administrativo y otros más. 
Después de hacer un recorrido por las tendencias de la educación superior en el nuevo siglo y tomando en cuenta el papel preponderante que juega la universidad, es indiscutible que el problema de la deserción no puede esperar más y urge enfocar recursos para buscar las causas particulares que originan esta situación.

Es importante recalcar que en los primeros años se da la etapa crítica, es aquí donde las autoridades de las instituciones educativas deben prestar especial atención a los cursos de orientación y de iniciación a la educación superior.

Es relevante tratar de descifrar el fenómeno de la deserción, no solo para determinar las causas específicas que llevan al estudiante a abandonar sus estudios, sino también determinar en qué medida la institución puede tomar acciones preventivas; pues es probable que el abandono no sea solo un problema personal del estudiante, sino que indique deficiencias del sistema en cuanto a orientación, apoyo y asistencia para los grupos más propensos a la deserción.

La magnitud del abandono refleja no sólo debilidades en la formación del estudiante que ingresa a las instituciones de Educación Superior, sino también debilidades en el sistema. Por ello se recomienda investigar más profundamente cuáles son las principales razones personales que mueven a un estudiante a tomar esta decisión, pues no basta con admitir estudiantes sino que hay que informarles $y$ educarlos en el sistema superior de enseñanza, para lograr en el futuro una relación más coherente entre el número de estudiantes admitidos y el número de estudiantes que culminan con éxito sus estudios. 


\section{Referencias bibliográficas}

Bolaños, Fernando (1985) Estudios de opinión a un grupo de 61 desertores de La UNED. San José: Editorial UNED.

García, Aretio, Lorenzo (1987) Rendimiento académico y abandono en la educación superior a distancia. Madrid: Editorial UNED

Granados, Tenorio (1992). Abandono de Estudios en las Facultades de letras de la UNED. Madrid: Simancas Ediciones S.A.

Jofré, Arturo.(1998) La universidad en América Latina. Cartago: Editorial Tecnológica de Costa Rica.

Ruiz, Ángel (2001). Educación superior en Costa Rica tendencias y retos en un nuevo escenario histónico. San José: Editorial UCR

UNED(1984) Foro Universitario. Perspectivas de la educación a distancia ante la crisis actual. San José: Editorial UNED. 